\title{
Synthesis of an Unsymmetrically Substituted, Dithienylethene-Containing 1,10-Phenanthroline Ligand and its Ruthenium(II) Complex
}

Joël Kühni, Vincent Adamo, Peter Belser*

Department of Chemistry, University of Fribourg, Chemin du musée 9, 1700 Fribourg, Switzerland

Fax +41(26)3009738; E-mail: Peter.Belser@unifr.ch

\begin{abstract}
The synthesis of an unsymmetrically substituted 1,10phenanthroline ligand and its corresponding ruthenium(II) complex is reported. Suzuki coupling has been employed under different conditions to get the target ligand.
\end{abstract}

Key words: metal complexes, cross-coupling, heterocycles, ligands, ruthenium

Organic dithienylethene derivatives have been extensively reported because of their potential applications in the field of photochromic systems and photonic devices. ${ }^{1}$ Metal complexes containing such derivatives as ligands are less common in literature. ${ }^{2}$ In general, new properties like energy transfer processes from a metal complex to an incorporated photochromic unit can be observed if a dithienylethene-substituted phenanthroline ligand is coordinated to a ruthenium complex. ${ }^{3}$ Recently, Yam et al. published a 1,10-phenanthroline ligand fused with a photochromic dithienylethene moiety and its rhenium(I) complex. ${ }^{4}$ In all the dithienylethene-phenanthroline systems reported so far, both thiophene moieties are identical. Here, we report the synthesis of an unsymmetrically substituted, dithienylethene containing 1,10-phenanthroline ligand 8 and its ruthenium(II) complex $\mathbf{R u}(\mathbf{8})$. The asymmetry was introduced by means of the two differently substituted thiophene units.

The thiophene moieties $\mathbf{3}$ and $\mathbf{5}$ were synthesized as shown in Scheme 1.

The precursor 3 was prepared from 2-isopropylthiophene $(\mathbf{1})^{5}$ in a two-step reaction. Compound $\mathbf{1}$ was brominated in chloroform-acetic acid (1:1) over five hours to form 3,5-dibromo-2-isopropylthiophene (2). $n$-BuLi was added to a solution of compound $\mathbf{2}$ in anhydrous THF at $-78{ }^{\circ} \mathrm{C}$ followed by the addition of tributyl borate. After warming to room temperature, $\mathrm{Pd}\left(\mathrm{PPh}_{3}\right)_{4}$, aqueous $\mathrm{Na}_{2} \mathrm{CO}_{3}$ $(20 \mathrm{wt} \%)$ and iodobenzene were added to the mixture to obtain 3-bromo-5-phenyl-2-isopropylthiophene (3) $(81 \%)$. The Suzuki coupling reaction was performed under deaerated conditions.

The precursor 5 was synthesized by reacting 3-bromo-2methyl-5-phenylthiophene $(4)^{6}$ with $n$-BuLi in THF at $-78{ }^{\circ} \mathrm{C}$. Then, tributyl borate was added to form the bo-
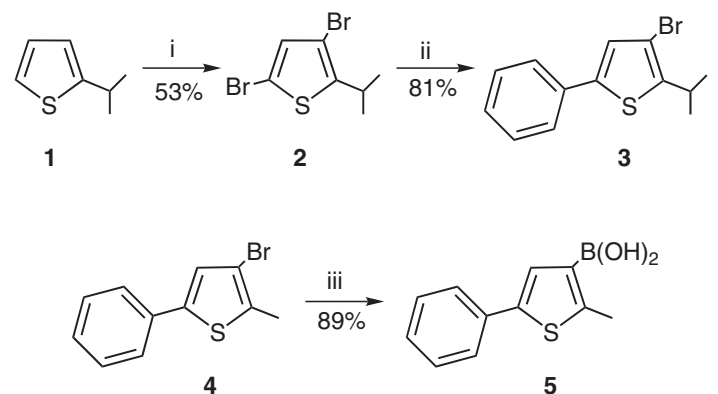

Scheme 1 Reagents and conditions: i) $\mathrm{Br}_{2}, 0{ }^{\circ} \mathrm{C}, \mathrm{CHCl}_{3}$-toluene (1:1); ii) $n$-BuLi, THF, $-78{ }^{\circ} \mathrm{C}$; tributyl borate; $\mathrm{PhI}, \mathrm{Pd}\left(\mathrm{PPh}_{3}\right)_{4}$, aq $\mathrm{Na}_{2} \mathrm{CO}_{3}, 70{ }^{\circ} \mathrm{C}, 24 \mathrm{~h}$; iii) $n$-BuLi, THF, $-78^{\circ} \mathrm{C}$; tributyl borate; concd $\mathrm{HCl}$.

ronic ester, which was hydrolyzed with hydrochloric acid to yield 5-phenyl-2-methylthio-3-boronic acid (5) .

Synthesis of ligand $\mathbf{8}$ was performed as described in Scheme 2. 5,6-Dibromo-1,10-phenanthroline (6) was prepared by the procedure outlined by Mlochowyski. ${ }^{7}$ $n$-BuLi was added to a solution of precursor 3 in anhyd THF at $-78{ }^{\circ} \mathrm{C}$ followed by the addition of tributyl borate. After warming to room temperature, Suzuki coupling was performed by adding $\mathrm{Pd}\left(\mathrm{PPh}_{3}\right)_{4}$ catalyst, aqueous $\mathrm{Na}_{2} \mathrm{CO}_{3}$ $(20 \mathrm{wt} \%)$ and 6 to furnish 5-bromo-6-(2-isopropyl-5thien-3-yl)-1,10-phenanthroline (7) in moderate yield. The homo disubstituted product, with two isopropyl sub-

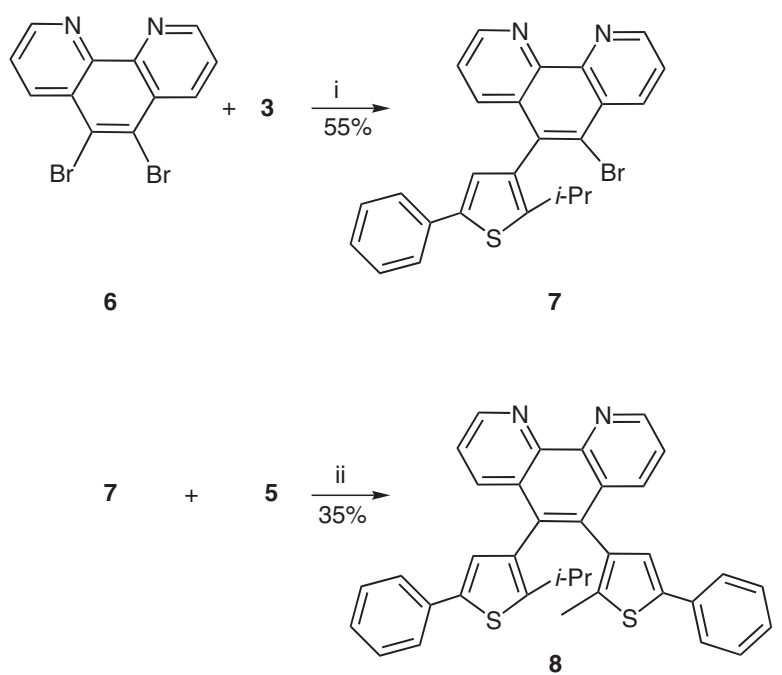

Scheme 2 Reagents and conditions: i) 3, $n$-BuLi, THF, $-78^{\circ} \mathrm{C}$; tributyl borate; 6, $\mathrm{Pd}\left(\mathrm{PPh}_{3}\right)_{4}$, aq $\mathrm{Na}_{2} \mathrm{CO}_{3}, 70^{\circ} \mathrm{C}$, overnight; ii) $\mathrm{Pd}\left(\mathrm{PPh}_{3}\right)_{4}$, aq $\mathrm{Na}_{2} \mathrm{CO}_{3}, 100{ }^{\circ} \mathrm{C}, 60 \mathrm{~h}$. 
stituents, was detected during the purification process in very poor yield (less than $1 \%$ ) and analyzed by ${ }^{1} \mathrm{H}$ NMR and MS. ${ }^{8}$ Compound 5 was dissolved in ethanol-toluene (1:1) and $\mathrm{Pd}\left(\mathrm{PPh}_{3}\right)_{4}$, aqueous $\mathrm{Na}_{2} \mathrm{CO}_{3}(20 \mathrm{wt} \%)$, and 7 was added to carry out a second Suzuki coupling reaction to get ligand $\mathbf{8}$ in moderate yield.

For the formation of the metal complex, $\left[\mathrm{Ru}(\mathrm{bpy})_{2}\right] \mathrm{Cl}_{2} \cdot 2 \mathrm{H}_{2} \mathrm{O}$ and ligand $\mathbf{8}$ were refluxed in methoxyethanol overnight. The solvent was removed and the residue was dissolved in water. The complex $\operatorname{Ru}(\mathbf{8})$ was precipitated with $\mathrm{NH}_{4} \mathrm{PF}_{6}$ and purified on silica gel preparative plate (Scheme 3 ).

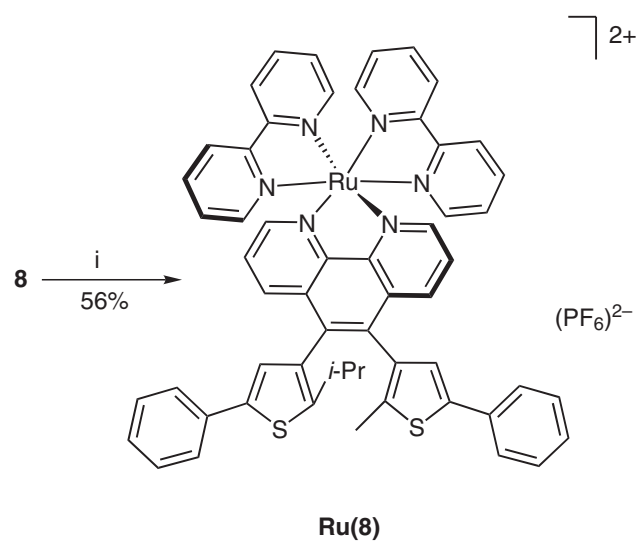

Scheme 3 Reagents and conditions: i) $\left[\mathrm{Ru}(\mathrm{bpy})_{2}\right] \mathrm{Cl}_{2} \cdot 2 \mathrm{H}_{2} \mathrm{O}$, methoxyethanol, reflux, $7 \mathrm{~h}$.

Ligand $\mathbf{8}$ and complex $\mathbf{R u}(\mathbf{8})$ showed no photochromism unlike the symmetrically substituted analogue with a methyl group attached to each of the two thiophene moieties. The reason lies in the exclusive formation of the syn-isomer of ligand $\mathbf{8}$ which is photo-inert and undergoes no photocyclization reaction. ${ }^{9}$

To conclude, we have developed a synthetic strategy, involving Suzuki coupling and manipulation of solvent polarity, to get the asymmetrically substituted ligand $\mathbf{8}$.

Column chromatography was performed using silica gel, 230-400 or 400-600 mesh size, from Chemie Brunschwig AG. Preparative plates $(20 \times 20 \mathrm{~cm})$ with silica gel $60 \mathrm{~F}_{254}$, having a layer thickness of $2 \mathrm{~mm}$ and aluminum sheets (for TLC) coated with silica gel 60 $\mathrm{F}_{254}$ were purchased from Merck.

All products were characterized by ${ }^{1} \mathrm{H}$ NMR and ${ }^{13} \mathrm{C}$ NMR spectra, recorded on a Bruker Avance DRX-400 (400.13 MHz, for ${ }^{1} \mathrm{H}$ NMR and $100.62 \mathrm{MHz}$ for ${ }^{13} \mathrm{C} \mathrm{NMR}$ ) and on a Bruker Avance DRX-360 spectrometer $\left(360 \mathrm{MHz}\right.$, for ${ }^{1} \mathrm{H}$ NMR and $90.55 \mathrm{MHz}$ for ${ }^{13} \mathrm{C}$ NMR). Chemical shifts are given in ppm using the solvent itself as internal standard. The chemical shifts are expressed as $\delta$ values and the coupling constants $(J)$ are given in Hertz.

Mass spectra were recorded on a HP 5988A Quadrupol (EI ionization, $70 \mathrm{eV}$ ) mass spectrometer. ESI and high-resolution mass spectra were recorded on a Bruker FTMS 4.7T BioAPEXII spectrometer.

GC-MS analyses were recorded on a ThermeQuest Finnigan VOYAGER GS/MS Trace GC 2000 Series equipped with an Optima-5MS column $(0.25 \mu \mathrm{m}, 25 \mathrm{~m} \times 0.32 \mathrm{~mm}$, Macherey-Nagel $)$.

\section{2,4-Dibromo-5-isopropylthiophene (2)}

2-Isopropylthiophene $(\mathbf{1} ; 2 \mathrm{~mL}, 16 \mathrm{mmol})$ was dissolved in $\mathrm{CHCl}_{3}$ toluene $(1: 1,10 \mathrm{~mL})$. The solution was cooled to $0{ }^{\circ} \mathrm{C}$ and $\mathrm{Br}_{2}$ (2.2 $\mathrm{mL}, 43 \mathrm{mmol})$ in $\mathrm{CHCl}_{3}$-toluene $(1: 1,5 \mathrm{~mL})$ was added dropwise over $5 \mathrm{~h}$. Stirring was continued overnight at r.t. $\mathrm{H}_{2} \mathrm{O}(20 \mathrm{~mL})$ was added to the dark brown solution and the aqueous phase was extracted with $\mathrm{CHCl}_{3}(3 \times 10 \mathrm{~mL})$. The combined organic fractions were neutralized $\left(\mathrm{Na}_{2} \mathrm{CO}_{3}\right)$, dried $\left(\mathrm{MgSO}_{4}\right)$ and filtered. The solvent was removed and the brown oil thus-obtained was purified by column chromatography (silica, hexane) to yield $\mathbf{2}$ as a colorless oil, yield: $2.3 \mathrm{~g}(53 \%)$.

IR (film): 3094, 2954, 2920, 2850, 1723, 1522, 1456, 1384, 1364, 1294, 1122, 1104, 1024, 966, 808, $806 \mathrm{~cm}^{-1}$.

${ }^{1} \mathrm{H} \mathrm{NMR}\left(\mathrm{CDCl}_{3}\right): \delta=6.97(\mathrm{~s}, 1 \mathrm{H}), 3.36(\mathrm{sept}, J=6.8 \mathrm{~Hz}, 1 \mathrm{H})$, $1.32(\mathrm{~d}, J=6.8 \mathrm{~Hz}, 1 \mathrm{H})$.

${ }^{13} \mathrm{C} \mathrm{NMR}\left(\mathrm{CDCl}_{3}\right): \delta=148.9,131.9,109.0,106.1,30.4,23.7$.

$\operatorname{MS}(\mathrm{EI}): m / z=283.9\left(\mathrm{M}^{+}\right)$.

\section{3-Bromo-2-isopropyl-5-phenylthiophene (3)}

To anhyd THF $(60 \mathrm{~mL})$ containing $2(2.5 \mathrm{~g}, 8.8 \mathrm{mmol})$, was added $n$-BuLi (1.6 M in hexane, $6 \mathrm{~mL}, 9.6 \mathrm{mmol}$ ) at $-78^{\circ} \mathrm{C}$ under argon, and the solution was stirred for $1 \mathrm{~h}$ at the same temperature. Tributyl borate $(3.5 \mathrm{~mL}, 13 \mathrm{mmol})$ was slowly added to the mixture and the stirring was continued for $1.5 \mathrm{~h}$ at $-78^{\circ} \mathrm{C}$. After warming up the solution to r.t., aq $20 \mathrm{wt} \% \mathrm{Na}_{2} \mathrm{CO}_{3}(22 \mathrm{~mL})$, iodobenzene $(1.8 \mathrm{~g}$, $1 \mathrm{~mL}, 8.8 \mathrm{mmol})$, and $\mathrm{Pd}\left(\mathrm{PPh}_{3}\right)_{4}(0.42 \mathrm{~g}, 0.38 \mathrm{mmol})$ were added. The mixture was refluxed at $70{ }^{\circ} \mathrm{C}$ for $24 \mathrm{~h}$. The product was extracted with $\mathrm{Et}_{2} \mathrm{O}$ and the organic fractions were dried $\left(\mathrm{MgSO}_{4}\right)$, filtered, and concentrated. The residue was purified by distillation (Kugelrohr-oven) to give 3 as a colorless oil; yield: $2.0 \mathrm{~g} \mathrm{(81 \% ).}$

IR (film): 3058, 3024, 2960, 2864, 1940, 1866, 1790, 1598, 1496, 1384, 1362, 1194, 1100, 1072, 1032, 1016, 952, 904, 866, 816, 754, $688 \mathrm{~cm}^{-1}$

${ }^{1} \mathrm{H}$ NMR $\left(\mathrm{CDCl}_{3}\right): \delta=7.59(\mathrm{~d}, 2 \mathrm{H}, J=7.7 \mathrm{~Hz}), 7.42(\mathrm{dd}, 2 \mathrm{H}$, $J=7.7,7.3 \mathrm{~Hz}), 7.34$ (t, $1 \mathrm{H}, J=7.3 \mathrm{~Hz}), 7.2$ (s, $1 \mathrm{H}), 3.44$ (sept, 1 $\mathrm{H}, J=6.8 \mathrm{~Hz}), 1.42(\mathrm{~d}, J=6.9 \mathrm{~Hz}, 6 \mathrm{H})$.

${ }^{13} \mathrm{C} \mathrm{NMR}\left(\mathrm{CDCl}_{3}\right): \delta=146.6,140.8133 .7,129.0,127.8,125.5$, 125.4, 107.5, 30.0, 23.9.

MS (EI): $m / z=281.9\left(\mathrm{M}^{+}\right)$

\section{2-Methyl-5-phenylthio-3-boronic Acid (5)}

$n$-BuLi (1.6 M in hexane, $6.2 \mathrm{~mL}, 9.9 \mathrm{mmol}, 1.1$ equiv) was added slowly to a stirred solution of 3-bromo-2-methyl-5-phenylthiophene (4; $2.27 \mathrm{~g}, 9.0 \mathrm{mmol}, 1$ equiv) in anhyd THF $(65 \mathrm{~mL})$ at $-78{ }^{\circ} \mathrm{C}$ under argon. After $60 \mathrm{~min}$, tributyl borate $(3.6 \mathrm{~mL}$, $13.5 \mathrm{mmol}, 1.5$ equiv) was added, and the stirring continued at $-78{ }^{\circ} \mathrm{C}$ for $5 \mathrm{~h}$ followed by warming up to r.t. After $15 \mathrm{~h}$, the mixture was added to $1.2 \mathrm{~N} \mathrm{HCl}(26 \mathrm{~mL})$ and stirred. The THF layer was separated and extracted with aq $1 \mathrm{~N} \mathrm{NaOH}(4 \times 7 \mathrm{~mL})$. The combined aqueous phases were then filtered to remove trace of solids, and then acidified to $\mathrm{pH} 1$ at $0{ }^{\circ} \mathrm{C}$ with conc. $\mathrm{HCl}$. The resulting precipitates were filtered, washed with $0.01 \mathrm{~N} \mathrm{HCl}$ and dried under vacuum, to yield 5 as a white powder; yield: $1.52 \mathrm{~g}(89 \%)$.

IR (KBr): 3336, 3060, 3022, 2970, 2862, 1932, 1858, 1780, 1598, 1540, 1498, 1466, 1438, 1378, 1308, 1286, 1178, 1120, 1046, 992, $848 \mathrm{~cm}^{-1}$.

${ }^{1} \mathrm{H}$ NMR (DMSO- $\left.d_{6}\right): \delta=2.60(\mathrm{~s}, 3 \mathrm{H}), 7.24(\mathrm{tt}, J=7.4,1.1 \mathrm{~Hz}, 1$ H), $7.38(\mathrm{dd}, J=7.4,8.3 \mathrm{~Hz}, 1 \mathrm{H}), 7.53(\mathrm{dd}, J=8.3,1.3 \mathrm{~Hz}, 1 \mathrm{H})$, 7.61 (s, $1 \mathrm{H}), 7.95$ (s, $2 \mathrm{H})$.

${ }^{13} \mathrm{C} \mathrm{NMR}\left(\mathrm{CDCl}_{3}\right): \delta=15.7,124.9,126.9,129.1,129.8,134.1$, 138.6, 148.6

MS (EI): $m / z=218.3\left(\mathrm{M}^{+}\right)$. 
5-Bromo-6-(2-isopropyl-5-phenylthien-3-yl)-1,10-phenanthroline (7)

To anhyd THF (3 mL) containing 3-bromo-2-isopropylthiophene (3) $120 \mathrm{mg}, 0.43 \mathrm{mmol}, 2$ equiv), was added $n$-BuLi (1.6 M in hexane, $0.3 \mathrm{~mL}, 0.48 \mathrm{mmol}, 2.25$ equiv) at $-78{ }^{\circ} \mathrm{C}$ under argon, and the resulting solution was stirred for $1 \mathrm{~h}$ at the same temperature. Tributyl borate $(0.2 \mathrm{~mL}, 0.74 \mathrm{mmol}, 3.5$ equiv) was slowly added to the mixture followed by stirring for another $1.5 \mathrm{~h}$ at $-78^{\circ} \mathrm{C}$. After warming the solution up to r.t., aq $20 \mathrm{wt} \% \mathrm{Na}_{2} \mathrm{CO}_{3}(1 \mathrm{~mL}), 5,6$-dibromophenanthroline $(\mathbf{6} ; 72 \mathrm{mg}, 0.21 \mathrm{mmol}, 1$ equiv $)$, and $\mathrm{Pd}\left(\mathrm{PPh}_{3}\right)_{4}(25 \mathrm{mg}, 0.021 \mathrm{mmol}, 10 \%)$ were added to the mixture and refluxed overnight at $70{ }^{\circ} \mathrm{C}$. The crude product was washed with $\mathrm{Et}_{2} \mathrm{O}$ and filtered to yield 7; yield: $113 \mathrm{mg}(58 \%)$.

IR (KBr): 3058, 2954, 2922, 2862, 1924, 1954, 1748, 1596, 1586, 1558, 1508, 1416, 1470, 906, 844, 802, 760, 738, $694 \mathrm{~cm}^{-1}$.

${ }^{1} \mathrm{H}$ NMR $\left(\mathrm{CDCl}_{3}\right): \delta=9.26(\mathrm{dd}, J=4.1,1.4 \mathrm{~Hz}, 1 \mathrm{H}), 9.23(\mathrm{dd}$, $J=4.5,1.4 \mathrm{~Hz}, 1 \mathrm{H}), 8.84(\mathrm{dd}, J=8.6,1.4 \mathrm{~Hz}, 1 \mathrm{H}), 8.01(\mathrm{dd}$, $J=8.2,1.4 \mathrm{~Hz}, 1 \mathrm{H}), 7.78(\mathrm{dd}, J=8.2,4.5 \mathrm{~Hz}, 1 \mathrm{H}), 7.64(\mathrm{~d}$, $J=7.7 \mathrm{~Hz}, 2 \mathrm{H}), 7.59(\mathrm{dd}, J=8.4,4.3 \mathrm{~Hz}, 1 \mathrm{H}), 7.38(\mathrm{dd}, J=7.5$, $7.5 \mathrm{~Hz}, 2 \mathrm{H}$ ), 7.21 (t, $J=7.2 \mathrm{~Hz}, 1 \mathrm{H}), 7.12$ (s, $1 \mathrm{H}$ ), 2.78 (sept, $J=6.8 \mathrm{~Hz}, 1 \mathrm{H}), 1.28(\mathrm{~d}, J=6.8 \mathrm{~Hz}, 3 \mathrm{H}) 1.12(\mathrm{~d}, J=6.8 \mathrm{~Hz}, 3 \mathrm{H})$.

${ }^{13} \mathrm{C} \mathrm{NMR}\left(\mathrm{CDCl}_{3}\right): \delta=151.2,150.9,149.5,141.8,137.9,137.3$, 135.5, 134.5, 133.3, 134.0, 129.9, 129.3, 129.1, 128.7, 127.8, 126.1, 125.6, 125.1, 124.4, 124.4, 124.1, 29.6, 25.3, 24.9.

MS (ESI): $m / z=461.1\left(\mathrm{M}^{+}+1\right)$.

HRMS: $m / z$ calcd for $\mathrm{C}_{25} \mathrm{H}_{20} \mathrm{BrN}_{2} \mathrm{~S}\left(\mathrm{M}^{+}+1\right)$ : 459.0525; found: 459.0525 .

6-(2-Isopropyl-5-phenylthien-3-yl)-5-(2-methyl-5-phenylthien3-yl)-1,10-phenanthroline (8)

A deaerated mixture of toluene $(2 \mathrm{~mL}), \mathrm{EtOH}(2 \mathrm{~mL})$ and aq $2 \mathrm{M}$ $\mathrm{Na}_{2} \mathrm{CO}_{3}(0.4 \mathrm{~mL})$ was added to a flask containing the boronic acid 5 (150 mg, $0.69 \mathrm{mmol}, 7.9$ equiv), bromophenanthroline 7 (40 mg, $0.087 \mathrm{mmol}, 1$ equiv) and $\mathrm{Pd}\left(\mathrm{PPh}_{3}\right)_{4}(130 \mathrm{mg}, 0.06 \mathrm{mmol}, 69 \%)$. Two immiscible phases were formed. The mixture was heated at $100{ }^{\circ} \mathrm{C}$ under argon for $60 \mathrm{~h}$ and then the solvents were removed in vacuo. The residual solid was further purified by column chromatography $\left(\mathrm{SiO}_{2}, \mathrm{CH}_{2} \mathrm{Cl}_{2}-\mathrm{Et}_{3} \mathrm{~N}, 100: 2\right.$, $)$ to give a white-brown solid, which was then washed with $\mathrm{Et}_{2} \mathrm{O}$ to afford the colorless product 8 ; yield: $17 \mathrm{mg}(35 \%)$.

IR (KBr): 3056, 3020, 2956, 2864, 1654, 1596, 1560, 1508, 1438, $1178,1118,808,746,722,692,540 \mathrm{~cm}^{-1}$.

${ }^{1} \mathrm{H} \mathrm{NMR}\left(\mathrm{CDCl}_{3}\right): \delta=1.01(\mathrm{~d}, J=6.8 \mathrm{~Hz}, 3 \mathrm{H}), 1.02(\mathrm{~d}, J=6.8 \mathrm{~Hz}$, $3 \mathrm{H}), 2.18$ (s, $3 \mathrm{H}), 2.64$ (sext, $J=6.8 \mathrm{~Hz}, 1 \mathrm{H}), 6.92$ (s, $1 \mathrm{H}), 7.20$ $(\mathrm{dd}, J=6.6,1.0 \mathrm{~Hz}, 2 \mathrm{H}), 7.21(\mathrm{~s}, 1 \mathrm{H}), 7.33(\mathrm{dd}, J=7.8,7.3 \mathrm{~Hz}, 2$ H), $7.39(\mathrm{dd}, J=7.5,7.5 \mathrm{~Hz}, 2 \mathrm{H}), 7.44-7.50(\mathrm{~m}, 1 \mathrm{H}), 7.60(\mathrm{~d}$, $J=7.1 \mathrm{~Hz}, 2 \mathrm{H}), 7.63-7.78(\mathrm{~m}, 3 \mathrm{H}), 8.18(\mathrm{~d}, J=8.3 \mathrm{~Hz}, 1 \mathrm{H}), 8.23$ $(\mathrm{d}, J=8.3 \mathrm{~Hz}, 1 \mathrm{H}), 9.26-9.36(\mathrm{~m}, 2 \mathrm{H})$.

${ }^{13} \mathrm{C} \mathrm{NMR}\left(\mathrm{CDCl}_{3}\right): \delta=14.6,24.5,26.1,29.0,125.6,125.6,125.8$, 126.5, 127.2, 127.4, 128.0, 128.4, 128.5, 128.8, 128.9, 129.4, 131.9, $131.9,132.0,132.1,132.5,133.0,133.6,134.2,134.3,134.8,136.2$, $140.5,140.6,149.9,149.9,151.1$.

MS (ESI): $m / z=553.18\left(\mathrm{M}^{+}+1\right)$.

HRMS: $m / z$ calcd for $\mathrm{C}_{36} \mathrm{H}_{29} \mathrm{~N}_{2} \mathrm{~S}_{2}\left(\mathrm{M}^{+}+1\right)$ : 553.1766; found: 553.1763 .

\section{Metal Complex Ru(8)}

To a 10-mL, one-necked, round-bottom flask were added the ligand 8 (15 mg, $27.2 \mu \mathrm{mol}, 1$ equiv), $\mathrm{Ru}(\mathrm{bpy})_{2} \mathrm{Cl}_{2} \cdot 2 \mathrm{H}_{2} \mathrm{O} \quad(15 \mathrm{mg}$, $29.9 \mu \mathrm{mol}, 1.1$ equiv) and methoxyethanol $(5 \mathrm{~mL})$. The solution was refluxed for $7 \mathrm{~h}$ at $120{ }^{\circ} \mathrm{C}$ under argon. The solvent was removed in vacuo and the residue was suspended in a solution of $\mathrm{NH}_{4} \mathrm{PF}_{6}(5 \mathrm{~mL}, 10 \mathrm{~g} / 150 \mathrm{~mL})$. The precipitate was collected by fil- tration and purified on a silica gel plate $\left(\mathrm{MeCN}-\mathrm{MeOH}-\mathrm{H}_{2} \mathrm{O} /\right.$ $\left.\mathrm{KNO}_{3}, 100: 10: 10: 1\right)$ to afford $\mathbf{R u}(\mathbf{8})$ as a red powder; yield: $19 \mathrm{mg}$ $(56 \%)$.

IR (KBr): 3076, 2958, 2922, 2852, 1704, 1660, 1602, 1466, 1446, $1426,838,762,558 \mathrm{~cm}^{-1}$.

${ }^{1} \mathrm{H}$ NMR $\left(\mathrm{CD}_{3} \mathrm{CN}\right): \delta=1.00-1.07(\mathrm{~m}, 3 \mathrm{H}), 1.09-1.15(\mathrm{~m}, 3 \mathrm{H})$, 1.00-1.07 (m, 3 H), 2.19-2.33 (m, 3 H), 2.71-2.85 (m, $1 \mathrm{H}), 2.90$ 3.07 (m, $1 \mathrm{H}), 7.05-7.29$ (m, $3 \mathrm{H}), 7.30-7.38$ (m, $3 \mathrm{H}), 7.39-7.46$ (m, $4 \mathrm{H}), 7.47-7.53(\mathrm{~m}, 3 \mathrm{H}), 7.55-7.61(\mathrm{~m}, 2 \mathrm{H}), 7.62-7.79$ (m, 5 $\mathrm{H})$, 7.87-7.92 (m, $2 \mathrm{H}), 8.03-8.10(\mathrm{~m}, 2 \mathrm{H}), 8.11-8.18$ (m, $4 \mathrm{H})$, 8.22-8.37 (m, $2 \mathrm{H}), 8.51-8.61(\mathrm{~m}, 4 \mathrm{H})$.

MS (ESI): $m / z=1111.18\left(\mathrm{M}^{+}-\mathrm{PF}_{6}^{-}\right)$.

HRMS: $m / z$ calcd for $\mathrm{C}_{56} \mathrm{H}_{44} \mathrm{~N}_{6} \mathrm{RuS}_{2}\left(\mathrm{M}^{2+}-2 \mathrm{PF}_{6}\right)$ : 483.1051; found: 483.1052 .

\section{Acknowledgment}

We thank the Swiss National Science Foundation for funding through the NFP47 program.

\section{References}

(1) (a) Tian, H.; Yang, S. Chem. Soc. Rev. 2004, 33, 85. (b) Irie, M. Chem. Rev. 2000, 100, 1685.

(2) (a) Adamo, V.; Belser, P. Chimia 2003, 57, 169. (b) Chen, B.; Wang, M.; Wu, Y.; Tian, H. Chem. Commun. 2002, 1060. (c) Fernandez-Acebes, A.; Lehn, J.-M. Adv. Mater. 1998, 10, 1519. (d) Fernandez-Acebes, A.; Lehn, J.-M. Chem. Eur. J. 1999, 5, 3285. (e) Fraysse, S.; Coudret, C.; Launay, J.-P. Eur. J. Inorg. Chem. 2000, 1581. (f) Jukes, R. T. F.; Adamo, V.; Hartl, F.; Belser, P.; De Cola, L. Inorg. Chem. 2004, 43, 2779. (g) Jukes, R. T. F.; Adamo, V.; Hartl, F.; Belser, P.; De Cola, L. Coord. Chem. Rev. 2005, 249, 1327. (h) Konaka, H.; Wu, L. P.; Munakata, M.; KurodaSowa, T.; Maekawa, M.; Suenaga, Y. Inorg. Chem. 2003, 42, 1928. (i) Matsuda, K.; Takayama, K.; Irie, M. Inorg. Chem. 2004, 43, 482. (j) Matsuda, K.; Takayama, K.; Irie, M. Chem. Commun. 2001, 363. (k) Munakata, M.; Wu, L. P.; Kuroda-Sowa, T. Bull. Chem. Soc. Jpn. 1997, 70, 1727. (1) Murguly, E.; Norsten, T. B.; Branda, N. R. Angew. Chem. Int. Ed. 2001, 40, 1752. (m) Samachetty, H. D.; Branda, N. R. Chem. Commun. 2005, 2840. (n) Sud, D.; McDonald, R.; Branda, N. R. Inorg. Chem. 2005, 44, 5960. (o) Takeshita, M.; Irie, M. J. Org. Chem 1998, 63, 6643. (p) Takeshita, M.; Irie, M. Tetrahedron Lett. 1998, 39, 613.

(3) Belser, P.; De Cola, L.; Hartl, F.; Adamo, V.; Bozic, B.; Iyer, Y. C. V. M.; Jukes, R. T. F.; Kühni, J.; Querol, M.; Roma, S.; Salluce, N. Adv. Funct. Mater. 2006, 16, 195.

(4) Yam, V. W.-W.; Ko, C.-C.; Zhu, N. J. Am. Chem. Soc. 2004, $126,12734$.

(5) Detty, M. R.; Hays, D. S. Heterocycles 1995, 40, 925.

(6) (a) Irie, M.; Lifka, T.; Kobatake, S.; Kato, N. J. Am. Chem. Soc. 2000, 122, 4871. (b) Kawai, S. H.; Gilat, S. L.; Ponsinet, R.; Lehn, J.-M. Chem. Eur. J. 1995, 1, 285. (c) Lantz, R.; Hornfeldt, A. B. Chem. Scr. 1972, 2, 9.

(7) Mlochowski, J. J. Rocz. Chem. 1974, 48, 2145.

(8) ${ }^{1} \mathrm{H} \mathrm{NMR}\left(\mathrm{CDCl}_{3}\right): \delta=9.34(\mathrm{~d}, J=4.1 \mathrm{~Hz}, 2 \mathrm{H}), 8.29(\mathrm{~d}$, $J=8.3 \mathrm{~Hz}, 2 \mathrm{H}), 7.71(\mathrm{dd}, J=8.3,4.3 \mathrm{~Hz}, 2 \mathrm{H}), 7.46(\mathrm{~d}$, $J=6.0 \mathrm{~Hz}, 4 \mathrm{H}), 7.30(\mathrm{dd}, J=7.3,6.1 \mathrm{~Hz}, 4 \mathrm{H}), 7.25(\mathrm{t}$, $J=7.1 \mathrm{~Hz}, 2 \mathrm{H}), 2.75$ (sept, $J=6.8 \mathrm{~Hz}, 2 \mathrm{H}), 1.18(\mathrm{~d}$, $J=6.8 \mathrm{~Hz}, 3 \mathrm{H}) 1.06(\mathrm{~d}, J=6.8 \mathrm{~Hz}, 3 \mathrm{H})$. MS (ESI+): $\mathrm{m} / \mathrm{z}=$ $581.2\left(\mathrm{M}^{+}+1\right)$.

(9) Kühni, J.; Adamo, V.; Belser, P. Chimia, submitted for publication. 